\title{
Educación y formación en emprendimiento social: características y creación de valor social sostenible en proyectos de emprendimiento social
}

\author{
Javier Sánchez Espada ${ }^{1}$, Sonia Martín López ${ }^{2}$, Paloma Bel Durán ${ }^{3}$ y Gustavo Lejarriaga \\ Pérez de las Vacas ${ }^{4}$
}

Recibido: 1 de agosto de 2018 / Aceptado: 14 de noviembre de 2018

Resumen. Tanto la OCDE, como la UE, se han mostrado proclives a la inclusión de la "iniciativa emprendedora" en las distintas etapas de la educación. En el año 2000 la formación para la adquisición de la cultura emprendedora se constituye como uno de los pilares centrales que deben guiar las políticas educativas de los países europeos. Con el RD 1147/2011, de 29 de julio, se introdujo el módulo profesional de empresa e iniciativa emprendedora. También la Ley 14/2013, de 27 de septiembre de apoyo a los emprendedores y su internacionalización, incorpora medidas para que los jóvenes adquieran, a través del sistema educativo, las competencias y habilidades requeridas para emprender.

Por otra parte, las instituciones europeas consideran "la promoción de la economía social como motor clave del desarrollo económico y social en Europa" (Resolución del Consejo Europeo de ministros de Empleo, Política Social, Sanidad y Consumidores de 7 de diciembre de 2015). El 23 de mayo de 2017 los Gobiernos de 11 países de la UE firmaron la 'Declaración de Madrid', en la que destacan la pertinencia de fomentar e incluir el emprendimiento bajo fórmulas de la economía social dentro de los planes de estudio en las diferentes etapas educativas.

El "emprendimiento social" está suscitando creciente interés, dada la necesidad de contribuir a generar valor a la misma dando respuesta a las necesidades sociales, es decir, tanto a los retos económicos, como sociales y medioambientales, buscando soluciones innovadoras, en línea con las prioridades de la Estrategia 2020 de la UE.

El presente artículo analiza las principales características exigibles a un proyecto de emprendimiento social, así como de la concreción de "la creación de valor sostenible", tanto en proyectos de emprendimiento social que cuentan con el reconocimiento de organizaciones de referencia, como en nuevos proyectos acometidos por jóvenes universitarios con base en las definiciones y rasgos diferenciadores que se obtienen como conclusión en los proyectos realizados por Cátedra de Emprendimiento Social UCM-Santander.

Palabras clave: Emprendimiento social; Creación de valor sostenible; Ecosistema emprendedor; Economía social.

1 Universidad Complutense de Madrid, España

Dirección de correo electrónico: javiersancchezespada@ucm.es

2 Universidad Complutense de Madrid, España

Dirección de correo electrónico: smartin@ucm.es

3 Universidad Complutense de Madrid, España

Dirección de correo electrónico: pbeldura@ucm.es

4 Universidad Complutense de Madrid, España

Dirección de correo electrónico: grlejarr@ucm.es 
Claves Econlit: P13; P19; L26; J54; M10.

[en] Education and training in social entrepreneurship: characteristics and creation of sustainable social value in social entrepreneurship projects

\begin{abstract}
OECD and the EU have have shown themselves to be inclined towards the inclusion of the " entrepreneurial initiative " in the various stages of education. In 2000, training for the acquisition of entrepreneurial culture became one of the central pillars that should guide the educational policies of European countries. With RD 1147/2011, of 29th July, the professional module for business and entrepreneurship was introduced. Law 14/2013, of 27 September, on support for entrepreneurs and their internationalisation, also includes measures for young people to acquire, through the education system, the skills and abilities required for entrepreneurship.

Moreover, the European institutions consider "the promotion of the social economy as a key driver of economic and social development in Europe" (Resolution of the European Council of Ministers for Employment, Social Policy, Health and Consumer Affairs of 7 December 2015). On 23 May 2017, the governments of 11 EU countries signed the 'Madrid Declaration', in which they stressed the relevance of promoting and including entrepreneurship under social economy formulas in the curricula at different stages of education.

Social entrepreneurship' is attracting increasing interest, given the need to help generate value by responding to social needs, i.e. economic, social and environmental challenges, by seeking innovative solutions in line with the priorities of the EU 2020 Strategy.

This paper analyzes the main characteristics required of a social entrepreneurship project, as well as the concretion of "the creation of sustainable value", both in social entrepreneurship projects that have the recognition of reference organizations, as in new projects undertaken by university students based on the definitions and differentiating features that are obtained as a conclusion in the projects carried out by the Cátedra de Emprendimiento Social UCM-Santander.

Keywords: Social entrepreneurship; Creation of sustainable value; Entrepreneurship Ecosystem; Social Economy.
\end{abstract}

Sumario. 1. Introducción. 2. Emprendimiento social, emprendimiento en economía social y creación de valor sostenible. 3. La concreción de "la creación de valor social sostenible": un estudio comparado. 4. Conclusiones. 5. Referencias bibliográficas.

Cómo citar: Sánchez Espada, J.; Martín López, S.; Bel Durán, P. y Lejarriaga Pérez de las Vacas, G. (2018) Educación y formación en emprendimiento social: características y creación de valor social sostenible en proyectos de emprendimiento social. REVESCO. Revista de Estudios Cooperativos, Tercer Cuatrimestre, No 129, pp. 16-38. DOI: 10.5209/REVE.62492.

\title{
1. Introducción
}

La economía social es un término que se tiene claramente definido en el ámbito de la Unión Europea. En un reciente estudio para el Comité Económico y Social Europeo (CESE), "la Economía Social en la Unión Europea"5, Chaves y Monzón la definen como "Conjunto de empresas privadas organizadas formalmente, con autonomía de decisión y libertad de adhesión, creadas para satisfacer las necesidades de sus socios a través del mercado, produciendo bienes y servicios, asegurando o financiando y en las que la eventual distribución entre los socios de beneficios o excedentes, así como la toma de decisiones no están ligados

\footnotetext{
5 Véase https://www.eesc.europa.eu/resources/docs/qe-30-12-790-es-c.pdf. Disponible en internet. Fecha de consulta: 25 de junio de 2018.
} 
directamente con el capital o cotizaciones aportados por cada socio, correspondiendo un voto a cada uno de ellos. La economía social también agrupa a aquellas entidades privadas organizadas formalmente con autonomía de decisión y libertad de adhesión que producen servicios de no mercado a favor de las familias, cuyos excedentes, si los hubiera, no pueden ser apropiados por los agentes económicos que las crean, controlan o financian” (Chaves y Monzón, 2012).

Los esfuerzos de clarificar este concepto vienen ya desde los años 90 del siglo pasado. De hecho, la anterior definición se basa en los criterios establecidos por el Manual de la Comisión Europea para la elaboración de las cuentas satélite de las empresas de la economía social y por (Barea, 1991; Barea y Monzón, 1995; Chaves y Monzón, 2000). Coincide tanto con los criterios delimitadores establecidos por las propias organizaciones de la economía social, carta del Comité Nacional de Enlace de las Actividades Mutualistas, Cooperativas y Asociativas (CNLAMCA) en el año 1980 y la Economía Social en Europa (SEE) en el año 2000 (Monzón y Chaves, 2017), como con las definiciones formuladas en la literatura económica, incluidos (Desroche, 1983; Defourny y Monzón, 1992; Defourny et al., 1999; Vienney, 1994; Demoustier, 2005).

Aún con todo, para muchos sigue siendo un término desconocido, mal utilizado y para algunos espuriamente asociado al tercer sector de forma exclusiva y excluyente, siendo ésta la tendencia reciente.

Pero la aceptación generalizada del término en Europa pone de manifiesto que seis países europeos ya han aprobado leyes sobre la economía social: Bélgica, España, Grecia, Portugal, Francia y Rumanía.

Las instituciones europeas consideran "la promoción de la economía social como motor clave del desarrollo económico y social en Europa" ${ }^{6}$ (Resolución del Consejo Europeo de ministros de Empleo, Política Social, Sanidad y Consumidores de 7 de diciembre de 2015). Se reconoce que "inspirándose en la fuerza de una larga tradición de economía social, los emprendedores sociales son motores de cambio y participan activamente para desarrollar y poner en práctica soluciones innovadoras a los principales retos económicos, sociales y medioambientales a los que se enfrenta la Unión Europea".

El 23 de mayo de 2017 los Gobiernos de 11 países de la Unión Europea firmaron la "Declaración de Madrid", por medio de la cual reivindican un fuerte impulso europeo de la Economía Social en la agenda política de la Unión Europea, al considerarla un modelo empresarial prioritario para un futuro sostenible en el que prime la cohesión social y económica. En esta declaración, acuerdan que las políticas nacionales y europeas de apoyo al emprendimiento deben tener en cuenta los modelos de la economía social como fórmula para la creación de un empleo inclusivo y una sociedad más justa, más equitativa y sostenible y que debe fomentarse la participación de la economía social en las actividades educativas, formativas y de capacitación profesional para la adquisición de competencias y el aprendizaje a lo largo de la vida. Destacan la pertinencia de fomentar e incluir el emprendimiento bajo fórmulas de la economía social dentro de los planes de estudio en las diferentes etapas educativas.

6 Véase http://www.observatorioeconomiasocial.es/media/archivos2012/ST_15071_2015_INIT_ES.pdf. 
Pues bien, las organizaciones de la economía social son una forma natural de emprender, particularmente en el sector de mercado (con las sociedades cooperativas y sociedades laborales como referente) con una demostrada aportación en épocas de crisis económicas y con unas características (no deslocalización, contribución al desarrollo, mayor compromiso y cohesión, entre otras) que las hacen atractivas como fórmulas generadoras de riqueza (Bel et al. 2016).

$\mathrm{Al}$ desconocimiento generalizado en materia de emprendimiento en general, se le añade el desconocimiento de la economía social en general y de las sociedades cooperativas en particular, lo que conlleva a la percepción negativa de estas formas jurídicas por parte de los nuevos emprendedores. Así lo ponen de manifiesto los estudios realizados desde la Universidad ${ }^{7}$. Las empresas de participación y de Economía Social son las grandes desconocidas, y esa falta de información provoca que las iniciativas de creación de sociedades no vayan dirigidas hacia las empresas sociales, aunque en épocas de crisis, son buenas generadoras de rentas y de mantenimiento de los niveles de empleo (Melián et al., 2017).

La Confederación Empresarial Española de la Economía Social (CEPES) ha sido sensible a esta circunstancia, firmando el 17 de abril de 2012 un convenio de colaboración con la Conferencia de Rectores de las Universidades Españolas (CRUE) en el contexto de una "jornada de sensibilización sobre la importancia del fomento del espíritu emprendedor de la economía social en las Universidades españolas" ". Es una iniciativa de interés que tendría que soportarse precisamente en las oficinas de apoyo a emprendedores de las disponen muchas de nuestras Universidades.

Se reconoce la obligación de la Universidad, como parte de su compromiso social, para favorecer y desarrollar un ecosistema emprendedor, que permita dar respuesta a los retos que presenta la sociedad, creación del empleo, así como, promover el emprendimiento social permitiendo construir un mundo mejor (Julia, 2013).

Es aquí donde los Universidades, oficinas técnicas y profesorado, pueden jugar un papel importante para difundir la cultura emprendedora, e incentivar el emprendimiento utilizando formas jurídicas enmarcadas dentro de la Economía Social (Martin et al., 2013).

Un estudio publicado en el año 2013 propone distintas opciones en el ámbito universitario que permiten fomentar el emprendimiento, cabe destacar las siguientes propuestas: organización de jornadas informativas para profundizar en el campo de la creación de empresas, asignaturas que permitan desarrollar habilidades para la creación de nuevos negocios, que finalizan con la presentación en eventos públicos de los proyectos, permitiendo la difusión de los mismos (Coque et al. 2013). En relación a la creación de un programa formativo, se plantea la necesidad de establecer un marco común que siente las bases de las competencias a desarrollar, metodología de la enseñanza, aprendizaje adecuado y sistemas de valoración que permitan evaluar los avances de los alumnos (Sáenz et al. 2015).

7 Ver: El emprendimiento individual (trabajo autónomo) como vía de acceso al mercado laboral por parte de los estudiantes y graduados universitarios. Proyecto presentado al Ministerio de Empleo y Seguridad Social, 2013.

8 Véase: http://www.crue.org/Documentos\%20compartidos/Convenios/Ref.195.pdf. 


\section{Emprendimiento social, emprendimiento en economía social y creación de valor sostenible}

No existe una interpretación consensuada en España del término emprendimiento y tampoco, por añadidura, del de emprendimiento social. El emprendimiento social, que suele atribuirse a William Drayton, hay quien lo concibe en un sentido restringido, limitándolo en ocasiones a entidades no ligadas al mercado, y quienes lo hacen en sentido amplio, incluyendo a organizaciones donde prima el interés social, ya sean de mercado o no (en línea con el concepto de economía social, de gran tradición en Europa).

Los emprendedores sociales, impactan positivamente en la Sociedad, ofreciendo bienes o servicios que cubren las necesidades que no han sido cubiertas por instituciones, y comparten un propósito principal, alcanzar fines sociales por encima de objetivos comerciales o financieras, obviamente sin incurrir en pérdidas, para garantizar la viabilidad y supervivencia de la empresa (Melián et al., 2017).

Desde la Cátedra de Emprendimiento Social UCM-Santander se ha desarrollado un proyecto encaminado a conocer la percepción del emprendimiento social por parte del profesorado universitario con el fin de proponer una definición del concepto que pueda contar con el mayor consenso posible, así como de identificar las características deseables y exigibles a un proyecto de emprendimiento social. Se parte de una definición inclusiva del concepto, esto es, se considera que emprender en cualquiera de las manifestaciones posibles de la economía social es emprendimiento social, y es por ello por lo que se solicitó al profesorado que se pronunciaran sobre diez de las definiciones de emprendimiento social más citadas en la literatura científica más una, la aportada por la Cátedra alineada a lo que se entiende por economía social.

Las definiciones propuestas son las siguientes:

- Aquella iniciativa que a través de fórmulas de mercado o no mercado contribuye a la cohesión social basándose en la primacía de las personas sobre el capital y con gobernanza democrática y/o participativa (definición propia basada en los conceptos de economía social y organizaciones de participación).

- Los emprendedores sociales son agentes de cambio importantes, cuyo núcleo de valores se centra en identificar, abordar y resolver problemas sociales (Drayton, 2002, p. 123).

- Asociaciones con fines sociales entre los sectores público, social y empresarial diseñadas para aprovechar el poder de mercado para el interés público. (Cook, et al. 2003, p. 66).

Proceso mediante el cual la creación de una nueva empresa de negocios conduce a la mejora de la riqueza social tanto para la sociedad como para el beneficio empresarial. (McMillan, 2003).

Organizaciones que han creado modelos para la resolución de manera eficiente de las necesidades humanas básicas que los mercados y las instituciones existentes no han podido satisfacer. El emprendimiento social combina el ingenio del espíritu empresarial tradicional con la misión de cambiar la sociedad. (Seelos y Mair, 2005, p. 241). 
- $\quad$ El emprendimiento social implica innovaciones destinadas a mejorar el bienestar social de forma explícita. Éste se encuentra dentro de organizaciones empresariales que inician, dirigen o contribuyen al cambio en la sociedad (Nichols, 2006, p. 2).

- $\quad$ El emprendimiento social es un tipo específico de emprendimiento que busca soluciones para problemas sociales a través de la construcción, evaluación y persecución de oportunidades que permitan la generación de valor social sostenible, alcanzando equilibrios nuevos y estables en relación con las condiciones sociales, a través de la acción directa llevada a cabo por organizaciones sin ánimo de lucro, empresas u organismos gubernamentales (Guzmán y Trujillo, 2008, p. 111).

El emprendimiento social es una actividad emprendedora con una orientación e intención social (Thompson, 2008, p. 157).

- $\quad$ El emprendimiento social abarca las actividades y procesos realizados para descubrir, definir y aprovechar las oportunidades para mejorar la riqueza social mediante la creación de nuevas empresas o la gestión de las organizaciones existentes de una manera innovadora (Zahra et al., 2009, p. 519).

- Los emprendedores sociales son personas con soluciones innovadoras a los problemas sociales más acuciantes de la sociedad. Son ambiciosos y persistentes, abordando los principales problemas sociales y ofreciendo nuevas ideas para el cambio a gran escala (Ashoka, 2014).

- El emprendimiento social se refiere a la práctica de combinar la innovación, el ingenio y la oportunidad de abordar los desafíos sociales y ambientales críticos. Los emprendedores sociales se centran en transformar los sistemas y prácticas que son las causas fundamentales de la pobreza, la marginación, el deterioro ambiental y la consiguiente pérdida de la dignidad humana. Al hacerlo, pueden configurarse con fines de lucro o sin fines de lucro, y en ambos casos, su objetivo principal es la creación de cambios sostenibles de los sistemas (Said Business School, 2014).

Del estudio referido, se concluye, que de entre las características deseables y exigibles a un proyecto de emprendimiento social, lo más destacado es la creación de valor social.

El valor social puede manifestarse de muy diferentes formas, con actuaciones respetuosas con el medio ambiente, favoreciendo la inclusión social, actuando de forma socialmente responsable, incidiendo sobre colectivos que requieren de una atención social no cubierta adecuadamente por el Estado o concretándose las iniciativas en fórmulas que contribuyen a la creación de empleo estable, al desarrollo territorial y a la cohesión social. Todo ello sin ánimo de exhaustividad (Lejarriaga y Bel, 2018).

Así, toda iniciativa, nuevo proyecto, en el que los promotores crean valor social en cualquiera de sus formas de concreción, se ha de considerar emprendimiento social.

El valor social, el interés social o cualquier otra de las características exigibles a un proyecto de emprendimiento social puede conseguirse a través de organizaciones empresariales o no empresariales. Si bien existen fórmulas jurídicas 
particularmente adaptadas a dar cabida a proyectos de emprendimiento social, estos pueden acogerse a cualquier modalidad legal posible (Martín, 2018). Sociedades cooperativas, sociedades laborales, asociaciones, fundaciones y mutualidades, todas ellas integrantes de la economía social, son formas naturales de emprendimiento social, pero, en términos generales, las organizaciones en las que los socios participan democráticamente en todos los flujos de la organización, en las que claramente se manifiesta la primacía de las personas sobre el capital porque los promotores fijan en democracia los objetivos y toman decisiones, suelen identificarse con proyectos de emprendimiento social. Así las cosas, las organizaciones de participación, que en términos generales incluyen a las entidades de la economía social, dan cabida también a proyectos sociales que se realizan bien individualmente (empresas individuales) o a determinadas sociedades con forma jurídica capitalista convencional.

La nueva definición surgida a raíz de la realización del estudio anteriormente citado propone la consideración de emprendimiento social a "Aquella iniciativa que a través de fórmulas de mercado o no mercado pretende la creación de valor social sostenible, bien por simple orientación e intención social o por su contribución a la cohesión social sobre la base de la primacía de las personas sobre el capital y su gobernanza democrática y/o participativa” (Lejarriaga y Bel, 2018). Esta definición la cumple cualquier organización de la economía social.

\section{La concreción de "la creación del valor social sostenible": un estudio comparado}

Con base en el estudio realizado previamente, se procura la descripción de las principales características exigibles a un proyecto de emprendimiento social, así como de la concreción de "la creación de valor sostenible", tanto en proyectos de emprendimiento social que cuentan con el reconocimiento de organizaciones de referencia, como en nuevos proyectos de emprendimiento social puestos en marcha por jóvenes universitarios.

La Universidad ha de cumplir las funciones que le requiere la sociedad basada en la adaptación de la formación superior al mercado del trabajo y a la posibilidad de transferir un conocimiento que fortalezca el tejido productivo a través del desarrollo de procesos de innovación empresariales. En este contexto la Universidad Complutense de Madrid se suma a las iniciativas de impulso y promoción del emprendimiento a través de la creación de una estructura específica, la Oficina Complutense del Emprendedor (Compluemprende).

Uno de los requerimientos que demanda nuestra sociedad, sin lugar a duda, es que la generación de riqueza que pueda producirse a través de emprendimiento contribuya a la creación de valor social.

Conscientes de esa misión, la Cátedra de Emprendimiento Social UCMSantander viene a reforzar el papel que desde hace mucho tiempo desarrollan, informalmente, la Oficina Complutense del Emprendedor y la Escuela de Estudios Cooperativos. Su misión: procurar que el conocimiento adquirido en nuestras aulas se transfiera a la sociedad a través de un emprendimiento socialmente responsable, democrático y participativo; esto es, a través del emprendimiento social. 
Para estudiar la concreción de la creación de valor social sostenible, tomaremos como referencia dos muestras:

- La primera muestra está formada por iniciativas de emprendimiento social reconocidas por entidades de referencia como es el caso de Ashoka, y por otro lado, se ha contado con la colaboración de ASALMA (Agrupación de Sociedades Laborales de Madrid) en la identificación de proyectos de emprendimiento social entre sus asociados.

- La segunda muestra, está formada por Proyectos Complutense considerados como proyectos de emprendimiento social que han participado en alguno de los programas o iniciativas de la Oficina Complutense del Emprendedor.

\subsection{Objetivos}

Analizar las principales características exigibles a un proyecto de emprendimiento social, así como de la concreción de "la creación de valor sostenible".

El estudio permitirá comparar los resultados con los que podrían derivarse de proyectos de emprendimiento social que cuentan con el reconocimiento de organizaciones de referencia.

\subsection{Metodología}

Para la recopilación de datos de la primera muestra se han seleccionado, por una parte, iniciativas de emprendimiento social reconocidas por entidades de referencia como es el caso de Ashoka, y por otra, se ha contado con la colaboración de ASALMA (Agrupación de Sociedades Laborales de Madrid) en la identificación de proyectos de emprendimiento social entre sus asociados.

Dicho proceso ha permitido la identificación de 47 proyectos de emprendimiento social, que son los que se han utilizado para la verificación del cumplimiento o no de las hipótesis planteadas.

En el caso de los Compluemprendedores, los datos se han obtenido a partir del envío de un cuestionario a los proyectos en fase inicial, en proceso de desarrollo y constituidos que han participado en las iniciativas desarrolladas por la Oficina Complutense del Emprendedor en los dos últimos años, entre los que se encuentran los presentados a la I Edición de los Premios de Emprendimiento Social promovidos por la Cátedra de Emprendimiento social. Se han seleccionado 38 proyectos.

Se establecen las siguientes hipótesis de partida que serán contrastadas en ambos colectivos:

Primera hipótesis: Las iniciativas de emprendimiento social se concretan en actuaciones respetuosas con el medio ambiente, favoreciendo la inclusión social, actuando de forma socialmente responsable, incidiendo sobre colectivos que requieren de una atención social no cubierta adecuadamente por el Estado o concretándose las iniciativas en fórmulas que contribuyen a la creación de empleo estable, al desarrollo territorial y a la cohesión social. 
- Segunda hipótesis: Los emprendedores sociales son más proclives a elegir fórmulas jurídicas integrantes de la economía social para poner en marcha sus iniciativas emprendedoras.

- Tercera hipótesis: Hay determinados sectores de actividad en los que se concentran en mayor medida iniciativas de emprendimiento social.

\subsection{Resultados}

\subsubsection{Hipótesis 1}

- Proyectos de emprendimiento social reconocido por las organizaciones representativas

Las principales manifestaciones de creación de valor social en proyectos de emprendimiento social reconocidos como tales por organizaciones de referencia como es el caso de Ashoka, son:

- La innovación social.

- La cohesión social.

- Las actuaciones socialmente responsables.

De las opciones propuestas están presentes en más del 50 por ciento de los casos; el favorecimiento de la innovación social (100 por cien), así como de la cohesión social (100 por cien), la actuación socialmente responsable (100 por cien), el favorecimiento de la concienciación social (91,43 por ciento), el favorecimiento del desarrollo territorial (74,29 por ciento), la creación de empleo estable (60 por ciento), el favorecimiento de la inclusión social (60 por ciento) y la incidencia sobre colectivos que requieren de una atención social no cubierta adecuadamente por el Estado (51,43 por ciento).

Por el contrario, las actuaciones respetuosas con el medio ambiente es el aspecto menos presente (tan sólo en el 34,29 por ciento de los casos según se pone de manifiesto en el Gráfico 1). 
Gráfico. 1. Manifestación de valor en proyectos de emprendimiento social reconocidos por organizaciones de referencia.

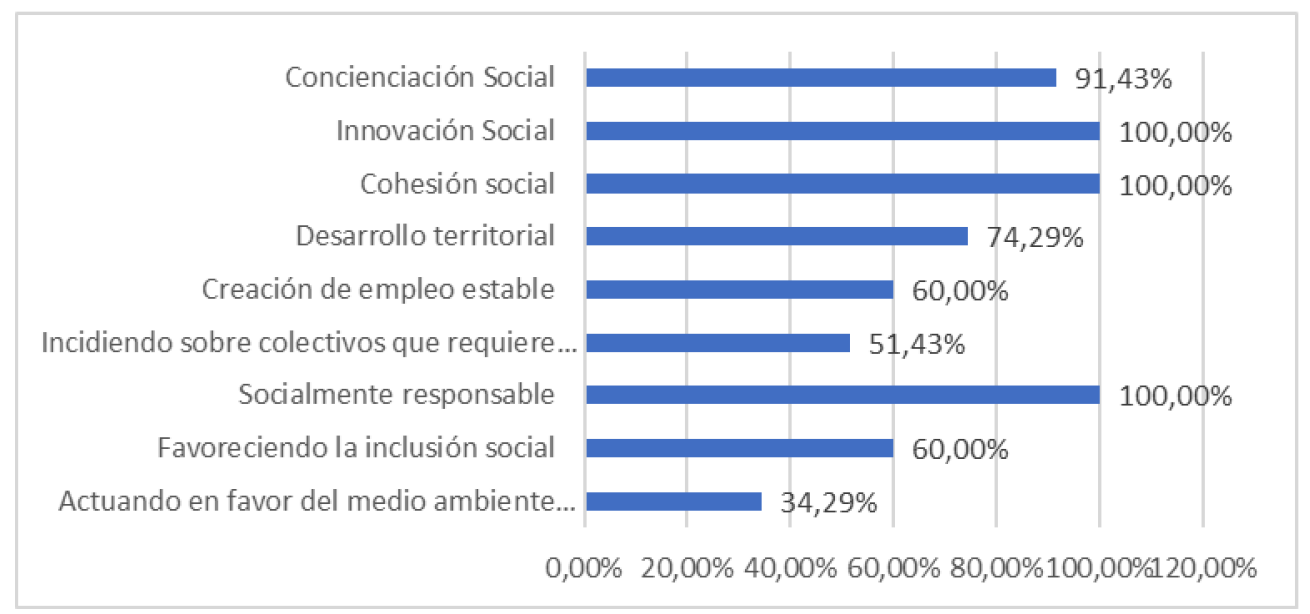

Fuente: Elaboración propia

En el caso de los proyectos de emprendimiento social miembros de ASALMA, de los que el 70 por ciento tienen la forma jurídica de Sociedad Limitada Laboral y el 30 por ciento restante de Sociedad Anónima Laboral, se obtiene que la creación de valor social en proyectos de emprendimiento social se manifiesta mediante el favorecimiento de la concienciación social, de la innovación social, así como la actuación socialmente responsable (100 por cien en todos los casos), el llevar a cabo actuaciones respetuosas con el medio ambiente (70 por ciento), el favorecimiento del desarrollo territorial (60 por ciento). Ver Gráfico 2.

Obtienen puntuaciones inferiores al 50 por ciento, el favorecimiento de la cohesión social, de la creación de empleo estable, de la inclusión social, así como la incidencia sobre colectivos que requieren de una atención social no cubierta adecuadamente por el Estado (40 por ciento, cada una de ellas). 
Gráfico. 2. Manifestación de valor en proyectos de emprendimiento social asociados a ASALMA.

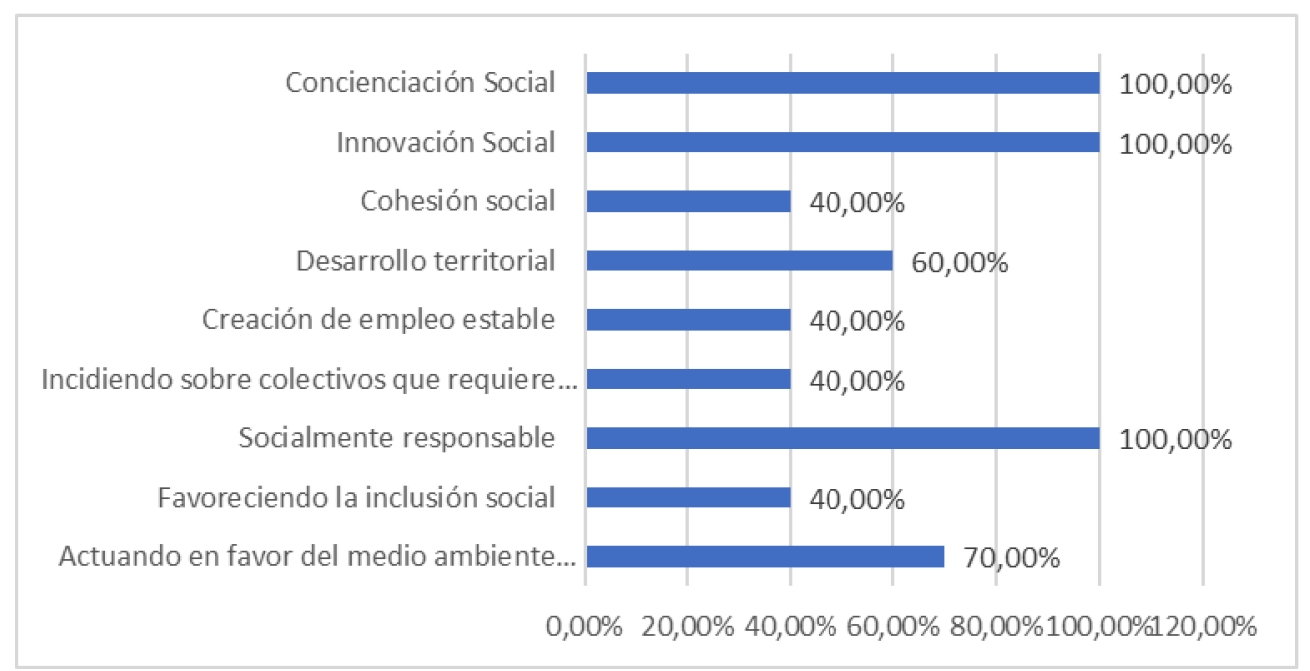

Fuente: Elaboración propia

\section{- Proyectos de emprendimiento social surgidos de la Universidad}

Se parte de la base de que los proyectos de emprendimiento activos que han participado en las iniciativas de la Oficina Complutense del Emprendedor, el 75 por ciento se autodefinen como sociales, cifra que se incrementa más aún si tenemos en cuenta los potenciales proyectos de emprendimiento alcanzando el 87,50 por ciento según se refleja en el Gráfico 3 . 
Gráfico. 3. Proyectos creados por Compluemprendedores que se autoidentifican de emprendimiento social.

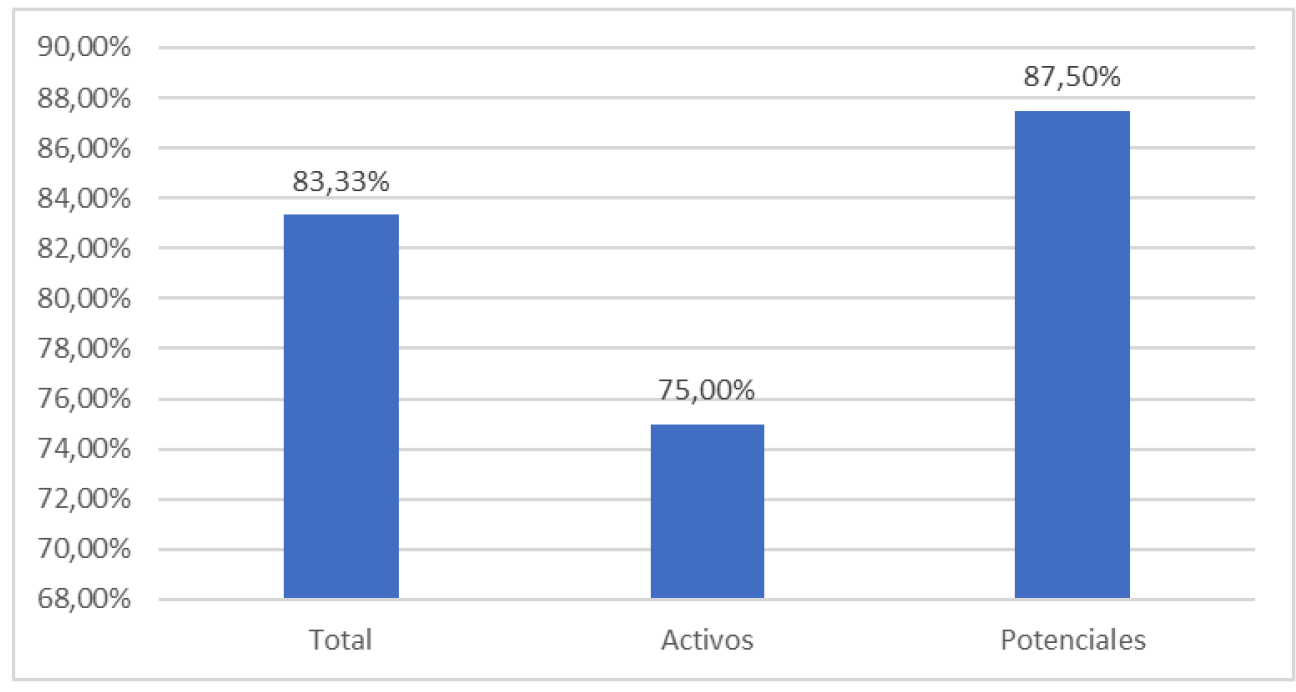

Fuente: Elaboración propia

En dichos proyectos, es la contribución al desarrollo local, la cohesión social y favorecimiento de colectivos con necesidad no cubierta las principales características manifestada (85 por ciento). En un porcentaje inferior, concretamente 31 y 23 por ciento, respectivamente, se representan las características relacionadas con el favorecimiento de la inclusión social y con acciones en favor del medio ambiente.

Gráfico. 4. Manifestación de valor en proyectos de emprendimiento social creados por Compluemprendedores.

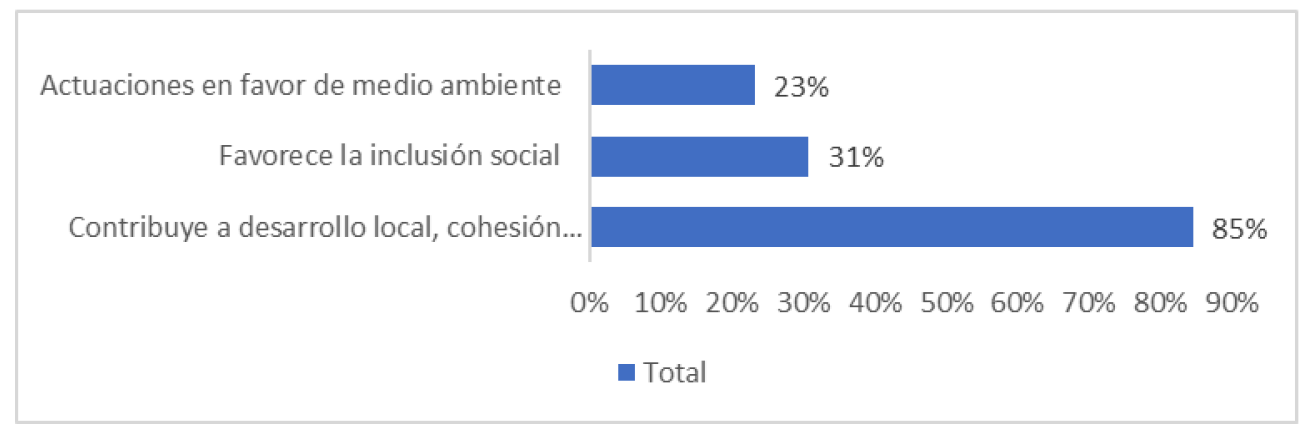

Fuente: Elaboración propia 


\subsubsection{Hipótesis 2}

La hipótesis 2 procura conocer en qué grado las fórmulas jurídicas relacionadas con la economía social son consideradas más adecuadas por los emprendedores sociales.

El estudio previo realizado captando información de los profesores sobre su conocimiento en torno al emprendimiento social, establece la siguiente distribución en cuanto a las formas jurídicas que más se ajustan a este tipo de iniciativas.

Gráfico. 5. Formas jurídicas que mejor se adaptan a emprendimientos sociales.

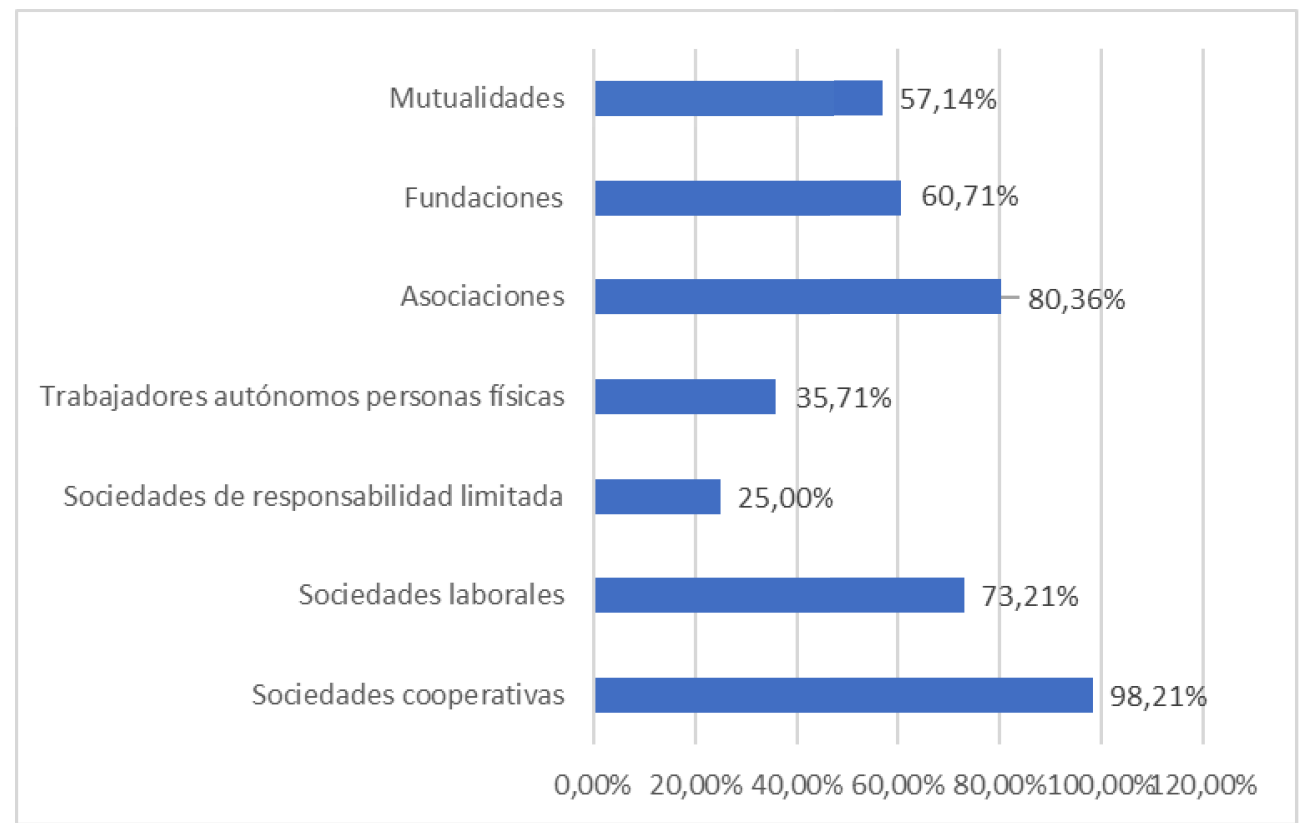

Fuente: Lejarriaga y Bel, 2018.

El contraste de la hipótesis se realiza a través de los dos colectivos señalados.

- Proyectos de emprendimiento social reconocido por las organizaciones representativas

En este caso, se pone de manifiesto que las formas jurídicas elegidas por los emprendedores sociales son las fundaciones ( 28,57 por ciento), las sociedades de responsabilidad limitada $\left(28,57\right.$ por ciento) y las asociaciones $(22,86 \text { por ciento })^{9}$.

9 Como no puede ser de otro modo, los proyectos de emprendimientos social asociados a ASALMA son sociedades anónimas y limitadas laborales y por tanto inmersas en la denominada economía social. 
Gráfico. 6. Forma jurídica de proyectos de emprendimiento social reconocidos por organizaciones de referencia.

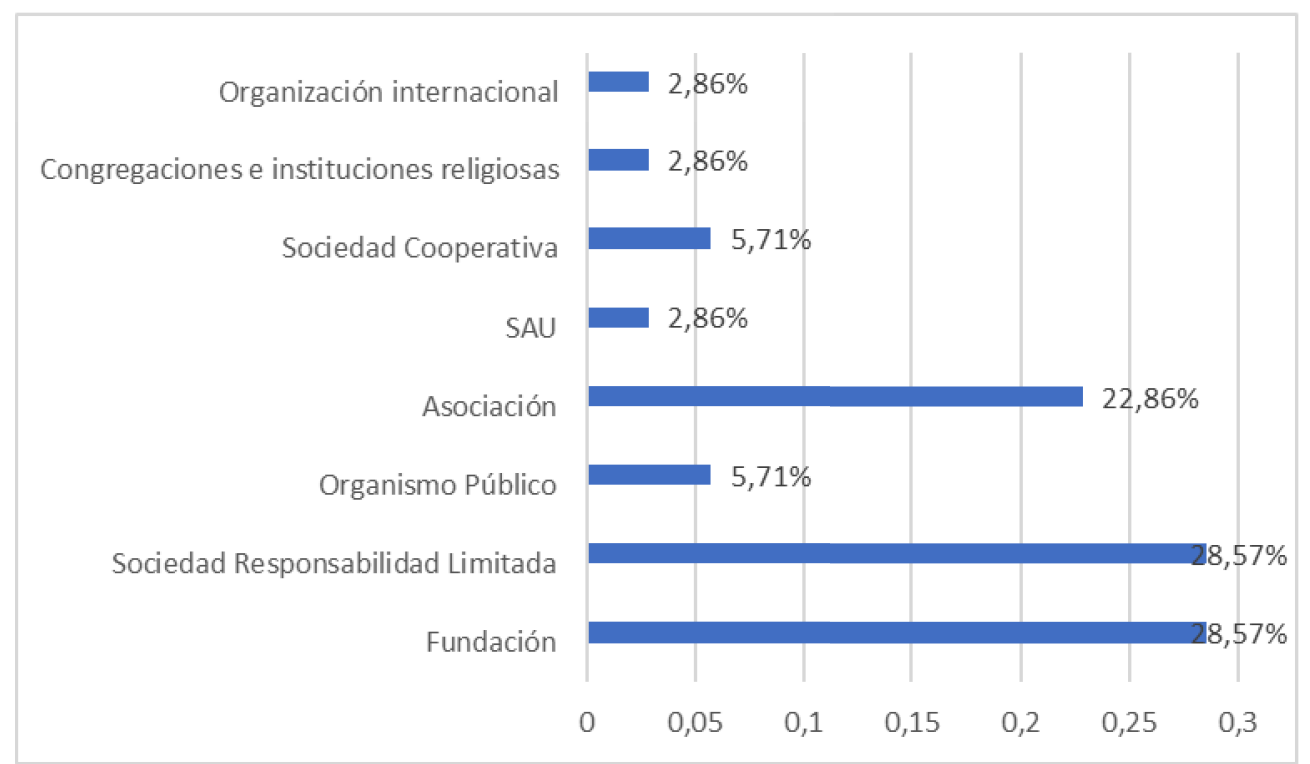

Fuente: Elaboración propia

\section{- Proyectos de emprendimiento social surgidos en la Universidad}

En el caso de los proyectos desarrollados por los Compluemprendedores se puede hacer una diferenciación entre las formas jurídicas que potencialmente podrían escoger los emprendedores para revestir sus proyectos y las que finalmente escogen cuando los ponen en marcha.

En el gráfico 7 se observa que las fórmulas jurídicas preferidas por los Compluemprendedores son las sociedades de responsabilidad limitada (41 por ciento) y trabajador por cuenta propia (33 por ciento) pero que, sin embargo, y aún con porcentajes minoritarios, tendrían en cuenta sociedades cooperativas, sociedades laborales y asociaciones. 
Gráfico. 7. Forma jurídica del total de proyectos creados por Compluemprendedores.

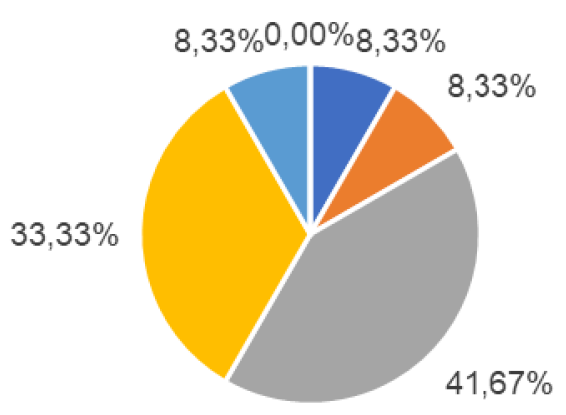

- Sociedad cooperativa

- Sociedad laboral

- Sociedad de responsabilidad limitada -" Trabajador autónomo persona física

- Asociación

- Fundación

Fuente: Elaboración propia

Sin embargo, cuando los proyectos son finalmente desarrollados se realiza un reparto equitativo entre sociedades de responsabilidad limitada y trabajadores autónomos y se prescinde de fórmulas jurídicas participativas o de la economía social.

Gráfico. 8. Forma jurídica elegida por proyectos creados por Compluemprendedores activos.

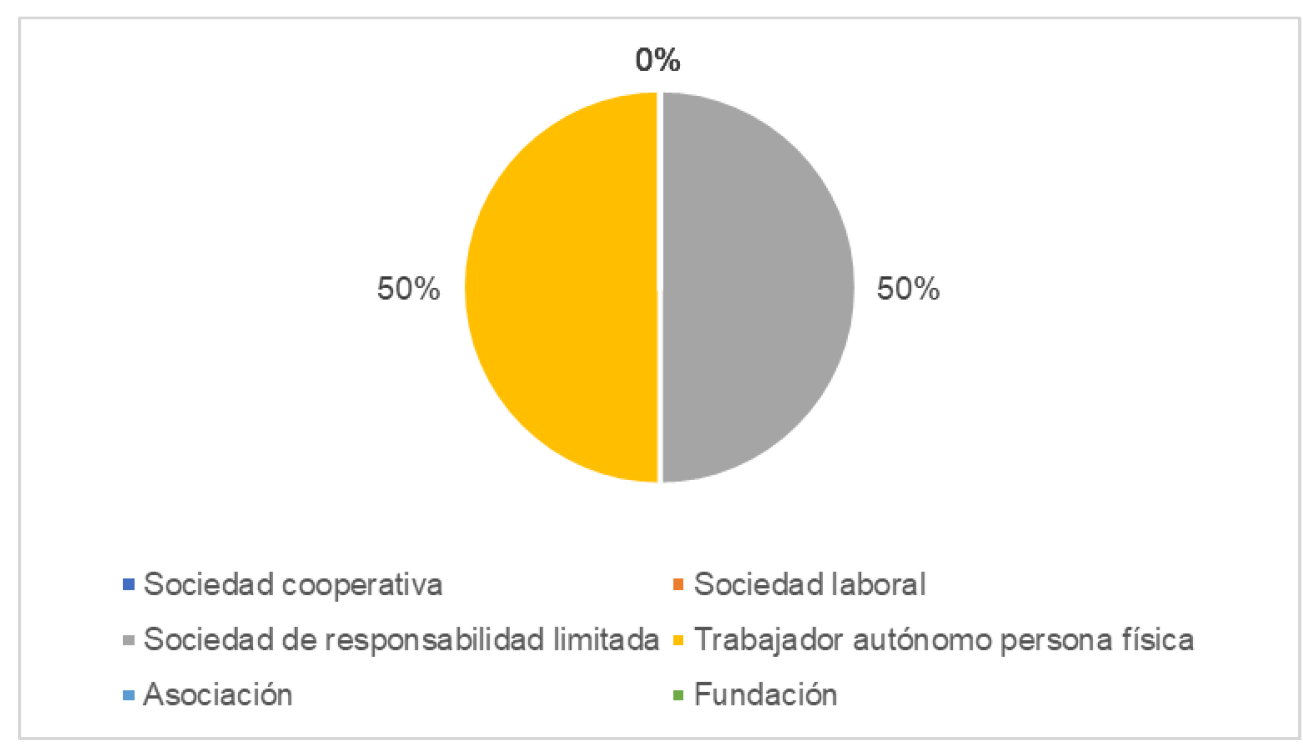

Fuente: Elaboración propia 
La explicación a dicha circunstancia puede encontrarse en las respuestas de los propios Compluemprendedores quienes desconsideran estas formas jurídicas, de una parte, porque se trata de proyectos unipersonales, y de otra, por la falta de asesoramiento y, derivado del mismo, el desconocimiento.

Gráfico. 9. Motivos por el que se desconsidera el resto de las formas jurídicas en proyectos Compluemprendedores.

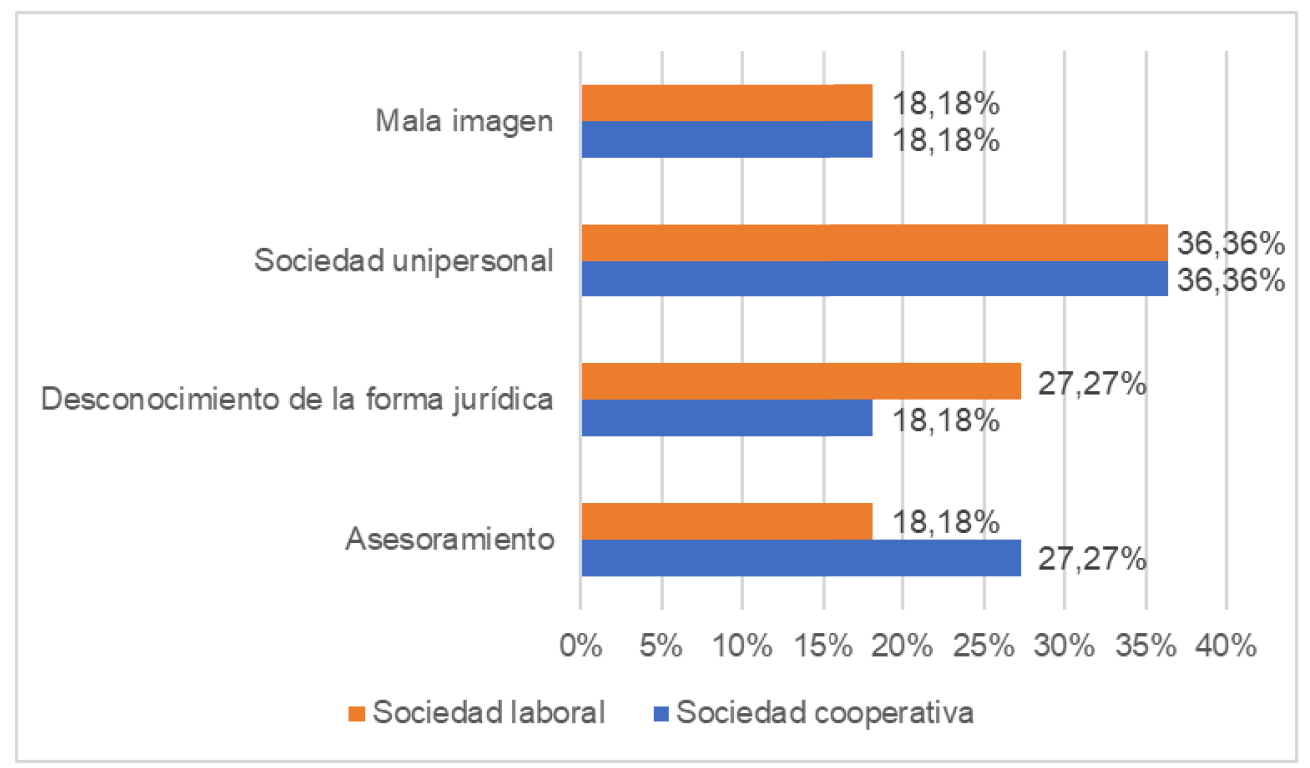

Fuente: Elaboración propia

\subsubsection{Hipótesis 3}

Hay determinados sectores de actividad que son más proclives a la concentración de proyectos de emprendimiento social.

\section{- Proyectos de emprendimiento social reconocido por las organizaciones representativas}

Del análisis de los sectores de actividad a los que pertenecen los proyectos de emprendimiento social analizados y reconocidos por organizaciones de referencia cabe destacar la presencia de iniciativas en el sector de la educación $(31,43$ por ciento), de la salud (20 por ciento), de los derechos humanos (17,14 por ciento), de medio ambiente (14,29 por ciento), así como de participación ciudadana, empleo y desarrollo económico (5,71 por ciento en cada uno de ellos). 
Gráfico. 10. Sector de actividad al que pertenecen proyectos de emprendimiento social reconocidos por organizaciones de referencia.

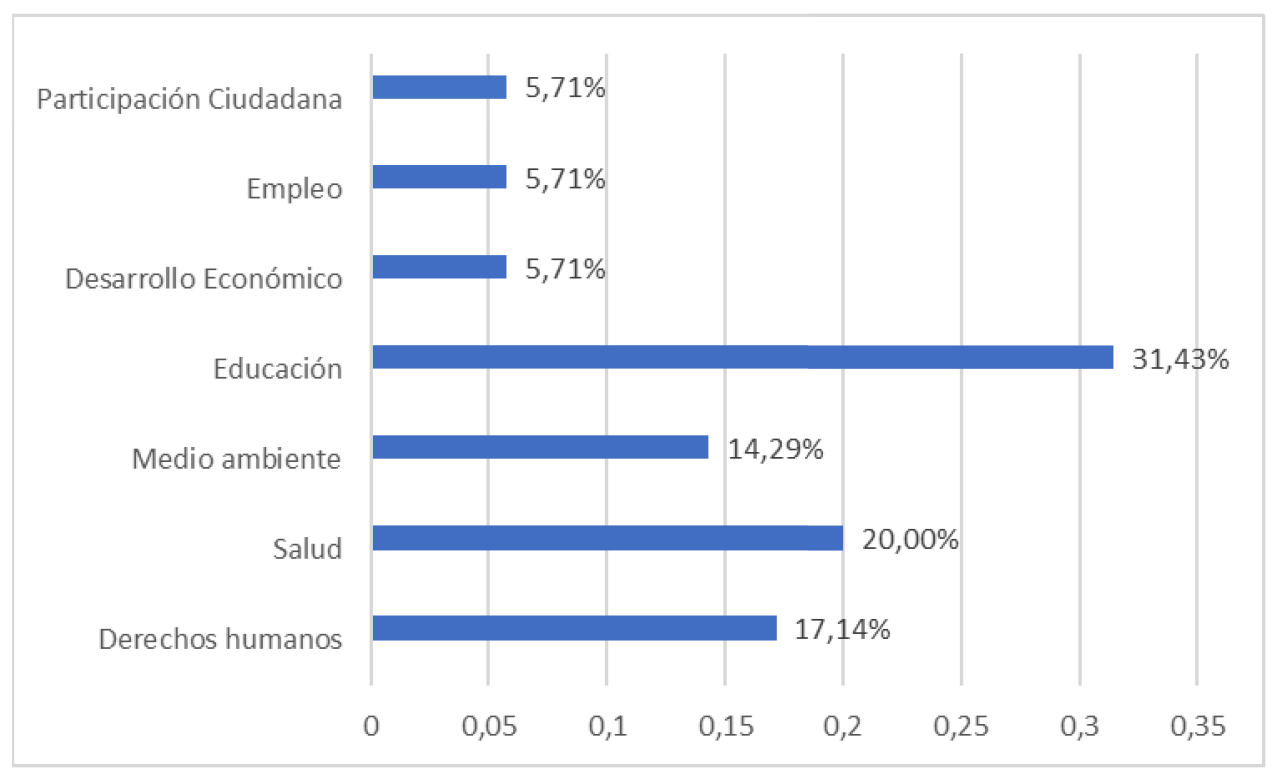

Fuente: Elaboración propia

Por lo que se refiere al análisis de los sectores de actividad a los que pertenecen los proyectos de emprendimiento social asociados a ASALMA, cabe destacar que pertenecen a sectores de actividad relacionados con las energías renovables (20 por ciento), la consultoría medioambiental (20 por ciento), la educación (20 por ciento), sector energético (10 por ciento), limpieza (10 por ciento), urbanismo (10 por ciento) y ayuda a domicilio (10 por ciento). 
Gráfico. 11. Sector de actividad al que pertenecen proyectos de emprendimiento social bajo la forma jurídica de Sociedad Laboral.

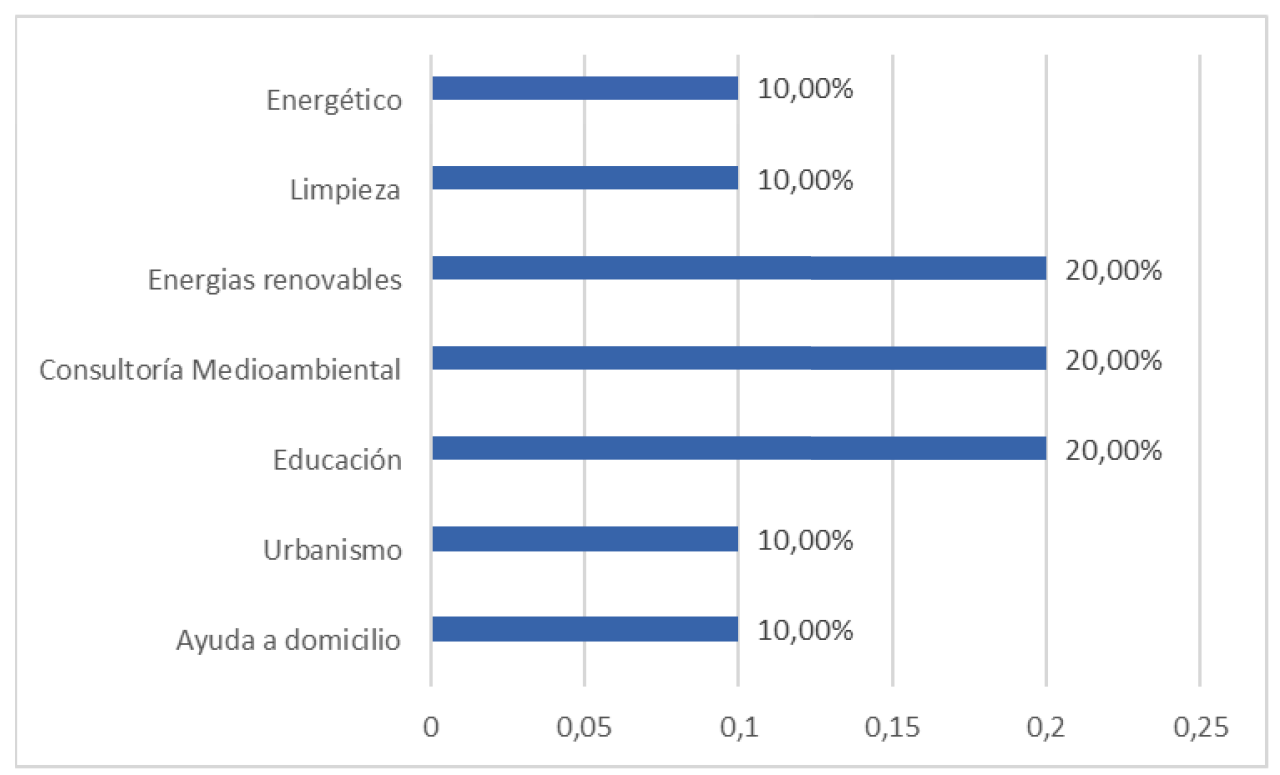

Fuente: Elaboración propia

\section{- Proyectos de emprendimiento social surgidos en la Universidad}

El 52,63 por ciento de los proyectos se engloban dentro del área de conocimiento de Ciencias Sociales, 23,68 por ciento dentro del área de Ciencias de la Salud, el 15,79 por ciento son proyectos que pertenecen al área de Arte y Humanidades. Por último, con un 5,26 y 2,63 por ciento encontramos las áreas de Ciencias e Ingeniería Informática respectivamente. 
Gráfico. 12. Área de conocimiento al que pertenecen los proyectos creados por Compluemprendedores.

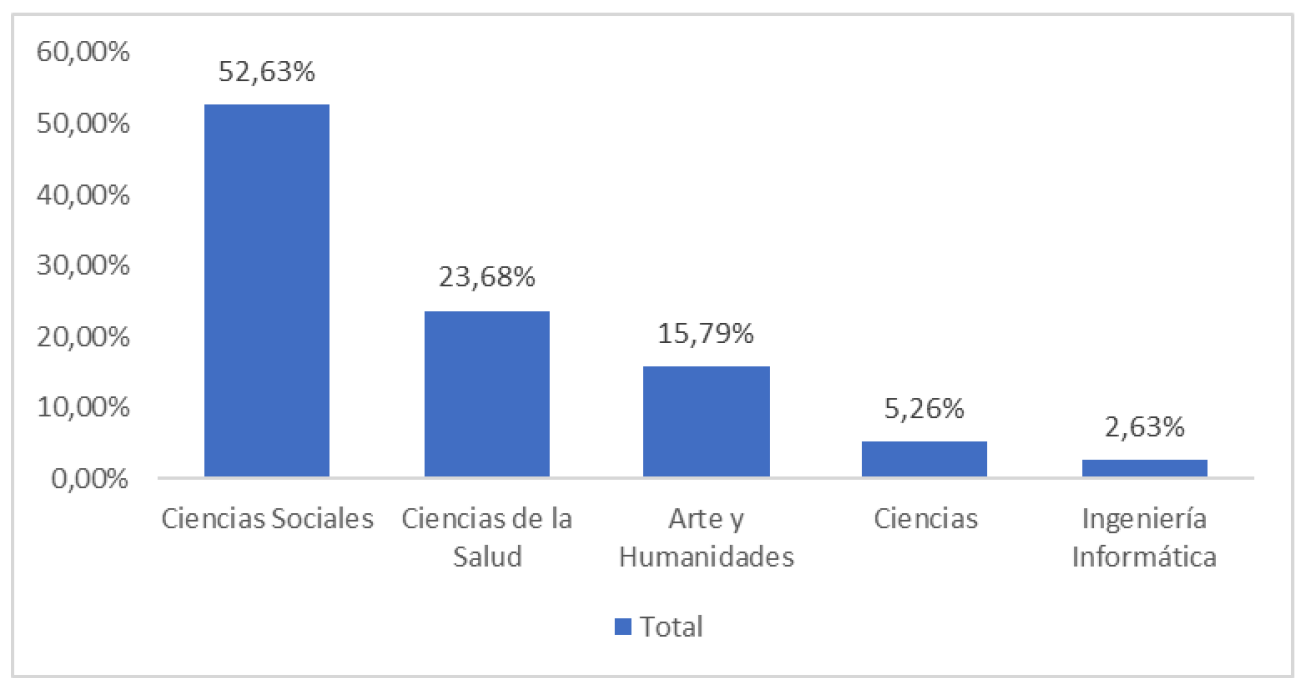

Fuente: Elaboración Propia

\section{Conclusiones}

El denominado "emprendimiento social" está suscitando creciente interés en la sociedad, dada la necesidad de contribuir desde el emprendimiento a generar valor a la misma dando respuesta a los problemas o necesidades sociales, es decir, tanto a los retos económicos, como sociales y medioambientales, buscando soluciones innovadoras.

Desde la Cátedra de Emprendimiento Social de la Universidad Complutense de Madrid se define el emprendimiento social como "Aquella iniciativa que a través de fórmulas de mercado o no mercado pretende la creación de valor social sostenible, bien por simple orientación e intención social o por su contribución a la cohesión social sobre la base de la primacía de las personas sobre el capital y su gobernanza democrática y/o participativa" (Lejarriaga y Bel, 2018).

La característica exigible a un proyecto de emprendimiento social que cuenta con mayor consenso entre los profesores, investigadores y emprendedores participantes en el estudio, con un 89,66 por ciento, es la creación de valor social.

El valor social en los proyectos de emprendimiento social puede manifestarse de muy diferentes formas (sin ánimo de exhaustividad): con actuaciones respetuosas con el medio ambiente, favoreciendo la inclusión social, actuando de forma socialmente responsable, incidiendo sobre colectivos que requieren de una atención social no cubierta adecuadamente por el Estado o concretándose las iniciativas en fórmulas que contribuyen a la creación de empleo estable, al desarrollo territorial y a la cohesión social.

Las principales manifestaciones de creación de valor social en proyectos de emprendimiento social, reconocidos como tales por organizaciones de referencia 
como es el caso de Ashoka, son el favorecimiento de la innovación social, de la cohesión social, así como la actuación socialmente responsable. Seguidas del favorecimiento de la concienciación social (91,43 por ciento), el favorecimiento del desarrollo territorial ( 74,29 por ciento), la creación de empleo estable (60 por ciento), el favorecimiento de la inclusión social (60 por ciento), la incidencia sobre colectivos que requieren de una atención social no cubierta adecuadamente por el Estado (51,43 por ciento) y llevar a cabo actuaciones respetuosas con el medio ambiente $(34,29$ por ciento).

En el caso de las Sociedades Laborales analizadas se consideran manifestaciones de creación de valor social en proyectos de emprendimiento social el favorecimiento de la concienciación social, de la innovación social, así como la actuación socialmente responsable (100 por cien en todos los casos), el llevar a cabo actuaciones respetuosas con el medio ambiente ( 70 por ciento), el favorecimiento del desarrollo territorial ( 60 por ciento). Obteniendo puntuaciones inferiores al 50 por ciento, el favorecimiento de la cohesión social, de la creación de empleo estable, de la inclusión social, así como la incidencia sobre colectivos que requieren de una atención social no cubierta adecuadamente por el Estado (40 por ciento, cada una de ellas).

Por otra parte, el estudio realizado desde la Cátedra de Emprendimiento Social de la Universidad Complutense de Madrid los docentes, investigadores y nuevos emprendedores participantes en el mismo, consideran casi de forma unánime que determinadas formas jurídicas, en términos generales, se adaptan mejor a los proyectos de emprendimiento social, siendo las sociedades cooperativas $(98,21$ por ciento), sociedades laborales $(73,21$ por ciento), asociaciones $(80,36$ por ciento) y fundaciones $(60,71$ por ciento) las que, de forma mayoritaria, se reconocen como formas naturales de acoger a este tipo de proyectos.

Del análisis de la forma jurídica de proyectos de emprendimiento social reconocidos por organizaciones de referencia se pone de manifiesto que las formas jurídicas elegidas por los emprendedores sociales son la fundación $(28,57$ por ciento), la sociedad de responsabilidad limitada $(28,57$ por ciento) y la asociación (22,86 por ciento).

Por tanto, aunque las sociedades cooperativas, sociedades laborales, asociaciones, fundaciones y mutualidades, todas ellas integrantes de la economía social, son formas naturales de emprendimiento social, hay otras formas jurídicas como las sociedades de responsabilidad limitada que no siendo de participación mantienen sus principales características: los socios participan democráticamente en todos los flujos de la organización y se manifiesta la primacía de las personas sobre el capital porque los promotores fijas en democracia los objetivos y toman decisiones. Éstas son las denominadas empresas de participación de hecho que suelen identificarse con proyectos de emprendimiento social lo que justifica la presencia de esta forma jurídica en las iniciativas desarrolladas y analizadas.

Del análisis de los sectores de actividad a los que pertenecen los proyectos de emprendimiento social analizados y reconocidos por organizaciones de referencia cabe destacar la presencia de iniciativas en el sector de la educación $(31,43$ por ciento), de la salud (20 por ciento), de los derechos humanos (17,14 por ciento), de medio ambiente $(14,29$ por ciento, así como de participación ciudadana, empleo y desarrollo económico (5,71 por ciento en cada uno de ellos). 
Por lo que se refiere al análisis de los sectores de actividad a los que pertenecen los proyectos de emprendimiento social analizados bajo la forma jurídica de Sociedades Laborales cabe destacar que pertenecen a sectores de actividad relacionados con las energías renovables ( 20 por ciento), la consultoría medioambiental (20 por ciento), la educación (20 por ciento), sector energético (10 por ciento), limpieza (10 por ciento), urbanismo (10 por ciento) y ayuda a domicilio (10 por ciento).

Por tanto, en ambos casos, se verifica la hipótesis de que los sectores de actividad relacionados con las manifestaciones consideradas previamente sobre creación de valor social son en los que están más presentes los proyectos de emprendimiento social.

Por su parte, de los estudios realizados podemos concluir que las iniciativas de emprendimiento social promovidas a través de la Universidad Complutense de Madrid son un reflejo de la realidad existente y analizada de otras iniciativas avaladas por organizaciones representativas y de las consideraciones realizadas por otros colectivos entrevistados como los profesores universitarios y los profesores de institutos de secundaria y formación profesional.

En este orden de cosas se destaca:

1. La cohesión social es una de las características más reconocida por todos los colectivos en su consideración de proyectos de emprendimiento social.

2. Los Compluemprendedores contemplan las mismas formas jurídicas que los profesores analizados para desarrollar futuros proyectos de emprendimiento social (asociaciones, fundaciones, trabajadores autónomos, sociedades cooperativas, sociedades laborales y sociedades de responsabilidad limitada). No obstante, cuando se disponen a emprender y a poner en práctica sus ideas sólo tienen en cuenta como traje jurídico a la sociedad de responsabilidad limitada y optan en el 50 por ciento de los casos por la figura del trabajador autónomo.

3. Los motivos son la falta de información y formación, y la falta de incentivos a la utilización de otros trajes jurídicos de participación.

4. En el sector educación se concentran un mayor número de proyectos de emprendimiento social.

5. Los Compluemprendedores, en general, se sienten emprendedores sociales y consideran entre sus objetivos la creación de empleo, la creación de valor social y la innovación.

6. Los Compluemprendedores no contemplan como característica identificativa del emprendimiento social la ausencia de ánimo de lucro, lo que se conviene con lo establecido por otros colectivos entrevistados y tampoco consideran importante la democracia, si bien en la mayoría de los proyectos que acometen tienen equidistribuido el capital social y, por tanto, se ejerce implícitamente la democracia.

El objetivo es dar continuidad a estos estudios incluyendo nuevas iniciativas de emprendimiento social que permitan ampliar la muestra, y con ello también el análisis llevado a cabo sobre las manifestaciones de creación de valor, permitiendo realizar análisis más detallados, tanto en función de la forma jurídica adoptada para 
poner en marcha los proyectos, como del objeto social y el sector de actividad en el que se encuadran.

\section{Referencias bibliográfícas}

Ashoka (2014) Todo el mundo puede ser impulsor de cambios. Disponible en internet: http://www.fundacionseres.org/Lists/ONLs/Attachments/15/Resumen\%20de\%20AShok a.pdf. Fecha de consulta: 13 de mayo de 2018.

Barea, J. (1991) La economía social en España. Revista de Economía y Sociología del Trabajo, $\mathrm{N}^{\circ} 12$, pp. 8-16.

Barea, J. y Monzón Campos, J.L. (1995) Las cuentas Satélite de la Economía Social en España; una primera aproximación. Valencia: Ciriec-España.

Bel Durán, P.; Fernández Guadaño, J.; Lejarriaga Pérez de las Vacas, G. y Martín López, S. (2016) La iniciativa emprendedora como base para la creación de empresas de participación. Un instrumento para la innovación social. Cooperativismo y Desarrollo, $\mathrm{N}^{\circ} 24$.

Chaves Ávila, R. y Monzón Campos, J.L. (2000) Las cooperativas en las modernas economías de mercado. Economistas, No 83, pp. 113-123.

Chaves Ávila, R. y Monzón Campos, J.L. (2012) Beyond the Crisis: The Social Economy, Prop a New Model of Sustainable Economic Development. Service Business, № 6, Vol. 1, p. 5-26.

Cook, B.; Dodds C.; Mitchell, W. (2003) Social Entrepreneurship: False Premises and Dangerous Forebodings. Australian Journal of Social Issues, $\mathrm{N}^{\circ} 38$, Vol. 1, pp. 57-72.

Coque Martínez, J.; Díaz Bretones, F. y López Mielgo, N. (2013) Factores para la puesta en marcha y el éxito de microempresas asociativas creadas por jóvenes egresados universitarios. REVESCO. Revista de Estudios Cooperativos, $\mathrm{N}^{\circ}$ 112, pp. 66-94. DOI: 10.5209/rev_REVE.2013.v112.43063.

Defourny, J. y Monzón Campos, J.L. (dir) (1992) Economía Social. Entre economía capitalista y economía pública. Valencia: Ciriec-España.

Defourny, J.; Develtere, P. y Fonteneau B. (1999) L'économie sociale au Nord et au Sud, De Boeck, Brussels.

Demoustier, D. (2005) Economie sociale et développement local, Cahiers de l'Economie Sociale, IES - París: L’Harmattan.

Desroche, H. (1983) Pour un traité d'Economie sociale, CIEM, París.

Drayton, W. (2002) The Citizen Sector: Becoming as Entrepreneurial and Competitive as Business. California Management Review, № 44, Vol. 3, pp. 120-132.

Guzmán Vázquez, A. y Trujillo Dávila, M. A. (2008) Emprendimiento social. Revisión de literatura. Estudios Gerenciales, № 24, Vol. 109, pp. 105-125.

Julià, J.F. (2013) Emprendimiento y Universidad. Una referencia al caso de España y la UPV. REVESCO. Revista de Estudios Cooperativos, Tercer Cuatrimestre, $\mathrm{N}^{\circ} 113$, pp. 7-27. DOI: 10.5209/rev_REVE.2014.v113.43394.

Lejarriaga Pérez de las Vacas, G. y Bel Durán, P. (2018) Emprendimiento, emprendimiento social, emprendimiento en economía social y emprendimiento a través de organizaciones de participación. Presentado en las V Jornadas de investigación y docencia en materia de empresas de participación. Emprendimiento social y nuevas formas de hacer economía: su relación con las organizaciones de participación y la economía social celebradas el 16 de febrero de 2018, en Madrid 
Mcmillan, I. A. (2003) Social Entrepreneurs: Playing the Role of Change Agents in Society. Disponible en internet: http://knowledge.wharton.upenn.edu/article/socialentrepreneurs-playing-the-role-of-change-agents-in-society/.

Martín López, S. (2018) ¿Cómo se concreta “la creación de valor social sostenible” en las iniciativas de emprendimiento social?. Ponencia presentada en el XII Congreso Internacional de Rulescoop. Brest, 22 al 24 de Mayo de 2018.

Martín López, S.; Fernández Guadaño, J.; Bel Durán, P. y Lejarriaga Pérez de las Vacas, G. (2013) Necesidad de medidas para impulsar la creación de las empresas de participación desde los niveles de enseñanza. Ciriec-España, Revista de Economía Pública, Social y Cooperativa, $\mathrm{N}^{\mathrm{o}} 78$, pp. 71-99.

Melián, A.; Campos, V. y Sanchis, J.R. (2017) La educación de postgrado en Economía Social en la universidad española ¿una asignatura pendiente. Ciriec-España, Revista de Economía Pública, Social y Cooperativa, No 89, pp. 33-54.

Melián, A; Campos, V. y Sanchis, J.R. (2011) Emprendimiento social y empresas de inserción en España. Aplicación del método Delphi para la determinación del perfil emprendedor y las empresas sociales creadas por emprendedores. REVESCO. Revista de Estudios Cooperativos, Tercer Cuatrimestre, No 106, pp. 150-172. DOI: 10.5209/rev_REVE.2011.v106.37377.

Monzón Campos, J.L. y Chaves Ávila, R. (2017) Evolución reciente de la economía social en la Unión Europea. Comité Económico y Social Europeo.

Nichols, A. (2006) Playing the Field: A New approach to the Meaning of Social Entrepreneurship. Social Enterprise Journal, No 2, Vol. 1, pp. 1-5.

Sáenz Bilbao, N. y López Vélez, A.L. (2015) Las competencias de emprendimiento social, COEMS: Aproximación a través de programas de formación universitaria en Iberoamérica. REVESCO. Revista de Estudios Cooperativos, Tercer Cuatrimestre, № 119, pp. 159-182. DOI: 10.5209/rev_REVE.2015.n119.49066.

Said Business School (2014) Defining Social Entrepreneurship. Disponible en internet: http://www.sbs.ox.ac.uk/ideas-impact/skoll/about-skoll-centre-socialentrepreneurship/what-social-entrepreneurship.

Seelos, C. y Mair, J. (2005) Social Entrepreneurship: Creating New Business Models to Serve the Poor. Business Horizons, № 48, Vol. 3, pp. 241-246.

Seres, fundación sociedad y empresa responsable. Disponible en: https://www.fundacionseres.org/Paginas/Campus/BuenasPracticas.aspx?IDe=34. Fecha de consulta: 13 de mayo de 2018.

Thompson, J. L. (2008) Social Enterprise and Social Entrepreneurship: Where Have We Reached?: a Summary of Issues and Discussion Points. Social Enterprise Journal, $\mathrm{N}^{\mathrm{o}} 4$, Vol. 2, pp. 149-161.

Vienney, C. (1994) L'Economíe sociale. Editions La Découverte, Paris.

Zahra, S. A.; Gedajlovic, E.; Neubaum, D. O. y Shulman, J. M. (2009) A Typology of Social Entrepreneurs: Motives, Search Processes and Ethical Challenges. Journal of Business Venturing, № 24, Vol. 5, pp. 519-532. 